\title{
Natural allelic variation of the IL-21 receptor modulates ischemic stroke infarct volume
}

\author{
Han Kyu Lee, ${ }^{1}$ Sehoon Keum, ${ }^{1}$ Huaxin Sheng, ${ }^{2}$ David S. Warner, ${ }^{2}$ Donald C. Lo, ${ }^{3}$ and Douglas A. Marchuk ${ }^{1}$ \\ 'Department of Molecular Cenetics and Microbiology, ${ }^{2}$ Department of Anesthesiology, and ${ }^{3}$ Center for Drug Discovery and Department of Neurobiology, Duke University Medical Center, \\ Durham, North Carolina, USA.
}

\begin{abstract}
Risk for ischemic stroke has a strong genetic basis, but heritable factors also contribute to the extent of damage after a stroke has occurred. We previously identified a locus on distal mouse chromosome 7 that contributes over $50 \%$ of the variation in postischemic cerebral infarct volume observed between inbred strains. Here, we used ancestral haplotype analysis to finemap this locus to 12 candidate genes. The gene encoding the IL-21 receptor (II21r) showed a marked difference in strain-specific transcription levels and coding variants in neonatal and adult cortical tissue. Collateral vessel connections were moderately reduced in I/21r-deficient mice, and cerebral infarct volume increased 2.3-fold, suggesting that II21r modulates both collateral vessel anatomy and innate neuroprotection. In brain slice explants, oxygen deprivation (OD) activated apoptotic pathways and increased neuronal cell death in IL-21 receptor-deficient (IL-21R-deficient) mice compared with control animals. We determined that the neuroprotective effects of IL-21R arose from signaling through JAK/STAT pathways and upregulation of caspase 3. Thus, natural genetic variation in murine I/21r influences neuronal cell viability after ischemia by modulating receptor function and downstream signal transduction. The identification of neuroprotective genes based on naturally occurring allelic variations has the potential to inform the development of drug targets for ischemic stroke treatment.
\end{abstract}

\section{Introduction}

Stroke is the fourth leading cause of death in the US, with almost 800,000 new cases occurring each year (1). The majority (over $80 \%$ ) of strokes are of ischemic origin, caused by an embolus or local thrombosis, leading to neural tissue damage (including infarction) in the territory of the occluded cerebral artery. Major risk factors (smoking, diabetes, obesity, etc.) for ischemic stroke have been well studied. A recent genome-wide complex trait analysis of human patients determined that heritability of ischemic stroke is $37.9 \%$, although there is wide variability between stroke subtypes (2). All of these studies have focused on genetic factors that modulate the ischemic stroke risk. These genetic risk factors contributing to the development of an ischemic stroke, especially those involving lipid metabolism, form the basis of current therapies to reduce ischemic stroke risk. However, genetic risk factors that modulate the extent of infarction once an ischemic stroke has occurred are largely unknown. These factors are difficult to identify in the human population due to uncontrollable variation in the extent and location of the occluded vessel and, especially, variation in the critical time between the first recognized symptoms of stroke and medical intervention. Decades of research on the molecular events occurring during infarction have failed to iden-

Note regarding evaluation of this manuscript: Manuscripts authored by scientists associated with Duke University, The University of North Carolina at Chapel Hill, Duke-NUS, and the Sanford-Burnham Medical Research Institute are handled not by members of the editorial board but rather by the science editors, who consult with selected external editors and reviewers.

Conflict of interest: The authors have declared that no conflict of interest exists. Submitted: August 31, 2015; Accepted: May 12, 2016.

Reference information: / Clin Invest. 2016;126(8):2827-2838. doi:10.1172/JCI84491. tify druggable targets to treat stroke. Therefore, we have taken an alternative, forward genetic (phenotype-driven) approach to discovering genes modulating the extent of infarction. Using surgical occlusion of the distal middle cerebral artery (MCAO) in 32 commonly used inbred mouse strains, we have previously shown that various inbred mouse strains demonstrate robust differences in infarct volume $(3,4)$. The differences in infarct volume are highly reproducible within each strain, but differ more than 50-fold between certain strains. Importantly, the differences are caused by natural allelic variation in the mouse genome. We have exploited these differences to map the natural genetic determinant of infarct volume to several quantitative trait loci (QTLs) that modulate the volume of cerebral infarction (3-5). One locus on distal chromosome 7, cerebral infarct volume QTL 1 (Civq1), contributes over $50 \%$ of the variation in infarct volume in multiple pairwise crosses of inbred mouse strains.

Although several pathophysiological mechanisms are thought to modulate cerebral infarct volume in human or animal models, recent studies have suggested that 2 major mechanisms, endogenous neuroprotection and pial collateral vessel anatomy, are the most important determinants of ischemic stroke infarct volume (6-9). Intriguingly, a locus on chromosome 7 that modulates variation in collateral vessel number (collateral artery number QTL 1 [Canq1]) (7) overlaps with Civq1. Two loci, Civq1 and Canq1, identified by mapping different but related phenotypes, share the same location on chromosome 7. Through multiple F2 intercrosses for both Civq1 and Canq1 loci and ancestral haplotype sharing, we have identified 12 candidate genes that might underlie one or both of these loci. In this present study, we demonstrate that natural allelic variation in IL-21 receptor (Il21r) modulates ischemic stroke infarct volume in mice through alteration of its receptor function. 
A

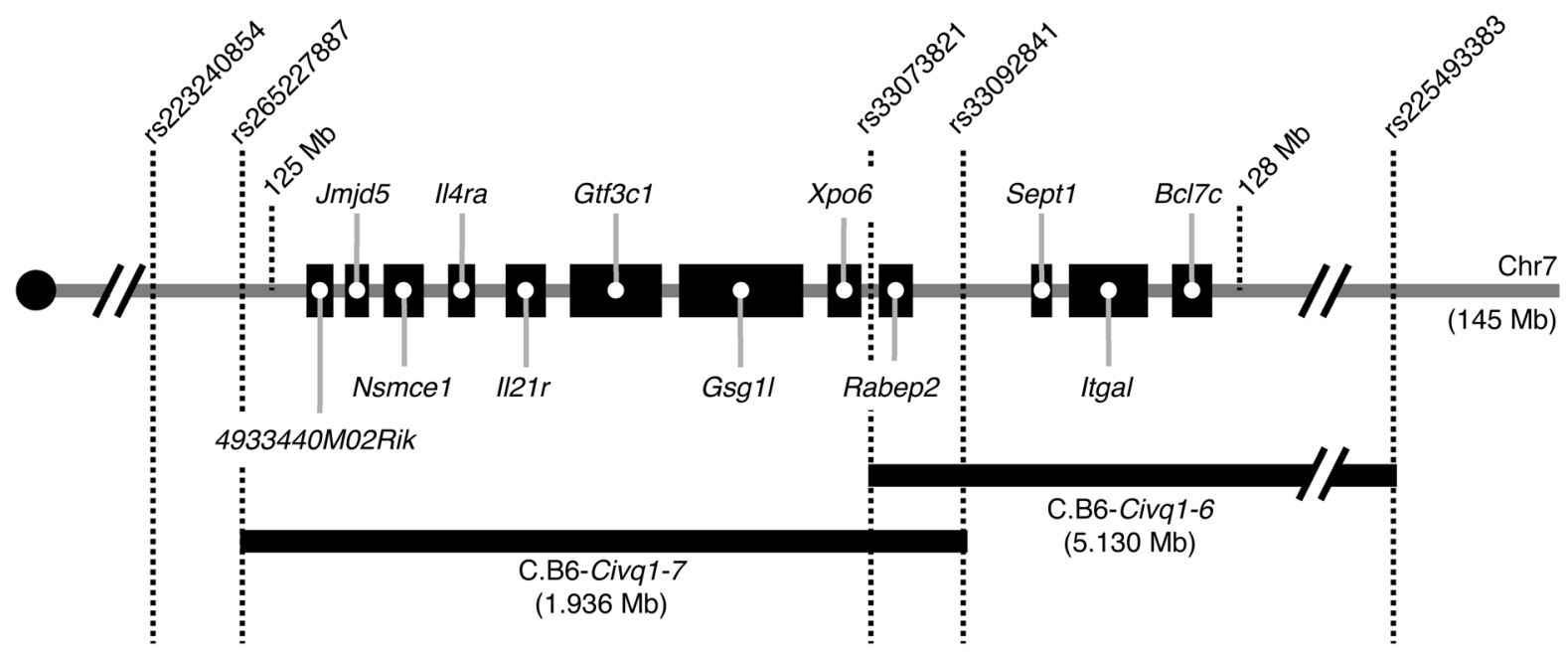

B

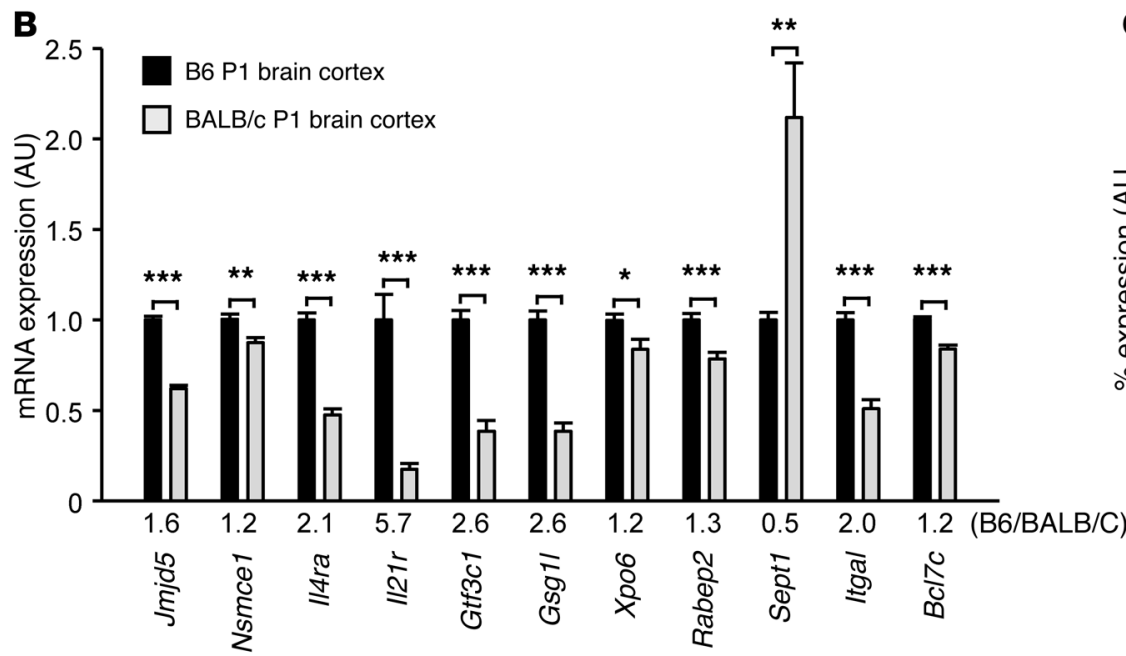

C

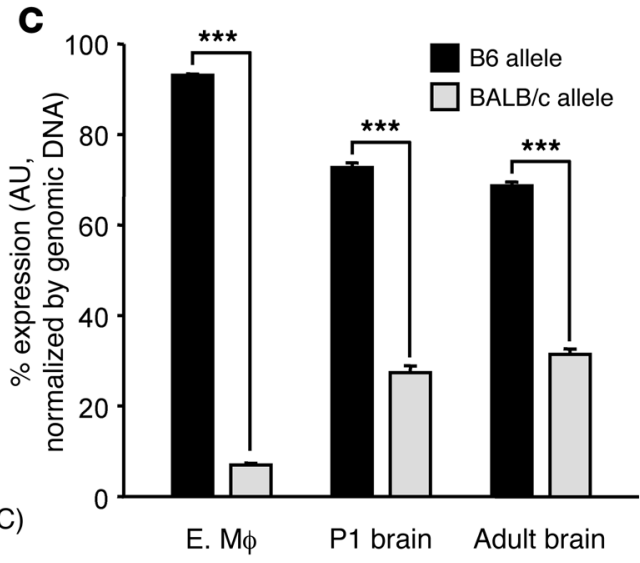

Figure 1. Candidate genes for Civq1 and Canq1 and their strain-specific transcript levels. (A) Schematic showing 12 candidate genes mapping within the Civq1 and Canq1 loci. Two Civq1 congenic lines, C.B6-Civq1-6 and C.B6-Civq1-7, carry segments of B6 chromosome 7 introgressed into the BALB/C background. Together, these 2 congenic strains cover the entire candidate region of Civq1. (B) Transcript levels of each gene transcript in P1 brain cortex from B6 and BALB/c mice were determined by qRT-PCR. Gapdh was used for normalization. Values represent mean \pm SEM from 7 B6 animals and 8 BALB/C animals. (C) The strain-specific mRNA expression level difference of the I/21r transcript. Graph represents allele-specific expression in tissues from F1 (B6-BALB/c) mice. A coding SNP (rs33008564) is used to monitor the allele-specific transcripts of II21r in embryonic macrophage (MФ) at E18.5, in P1 brain cortex, and in adult brain cortex. Genomic DNA was used for normalization. Values represent mean \pm SEM from at least 3 animals per tissue (6 animals for embryonic macrophage, 5 animals for P1 brain cortex, and 3 animals for adult brain cortex). ${ }^{*} P<0.05 ;{ }^{* *} P<0.01$; ${ }^{* *} P<0.001$, 2-tailed Student's $t$ test.

\section{Results}

Fine-mapping of both Civq1 and Canq1 identifies candidate genes. Previously, we identified a locus on distal chromosome 7 (Civq1) as a major determinant of infarct volume after permanent MCAO $(3,4)$. Civq1 overlaps a locus modulating cerebral collateral vessel number, Canq1 (7). Zhang et al. also demonstrated that pial collateral vessel anatomy in the brain is inversely correlated with infarct volume (7). This correlation might imply that allelic variation in the same gene or genes modulates the phenotypes of both infarct volume and pial collateral vessel density in the brain. However, infarct volume represents a phenotypic outcome that can include effects due to many different endophenotypes, including reperfusion due to collateral circulation, endogenous neuroprotection, inflammation, and other mechanisms. Thus, the Civq1 locus is likely to include the gene or genes underlying the overlapping Canq1 locus, but could also include additional genes acting via other (e.g., collateral-independent) mechanisms. We sought to identify a gene or genes for Civq1, mindful that at least some of these would be related to collateral vessel anatomy, but being aware that the same or different genes with other functional mechanisms may also be in play.

To further refine the list of potential candidate genes, we employed shared (ancestral) haplotype analysis, being careful to include only inbred strains for which we had generated genetic linkage data that supported the existence of Civq1 (or Canq1) in an intercross between 2 inbred mouse strains. We had identified Civq1 (infarct volume) in 3 different crosses between inbred strains C57BL/6 (B6) $\times$ BALB/c, B6 $\times$ SWR, and FVB $\times$ BALB $/ \mathrm{c}$ $(3,4)$. The Canq1 locus (collateral vessel number) was previously identified in a B6 $\times$ BALB/c intercross (10). For this study, we mapped Canq1 (collateral vessel number) in 3 additional intercrosses: $\mathrm{B} 6 \times \mathrm{A} / \mathrm{J}, \mathrm{FVB} \times \mathrm{BALB} / \mathrm{c}$, and NOD $\times \mathrm{BALB} / \mathrm{c}($ Supple- 


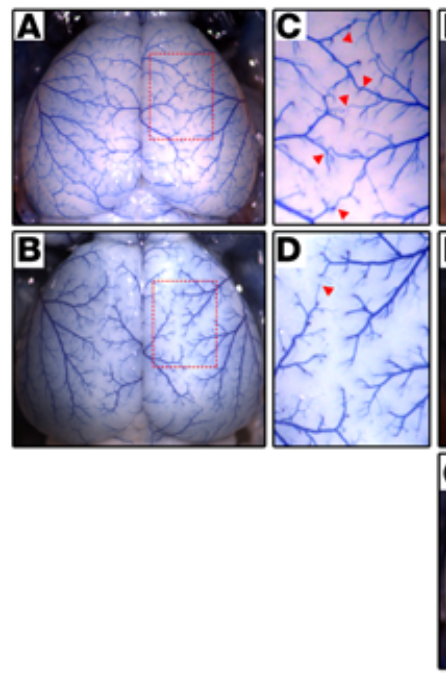

$\mathbf{K}$
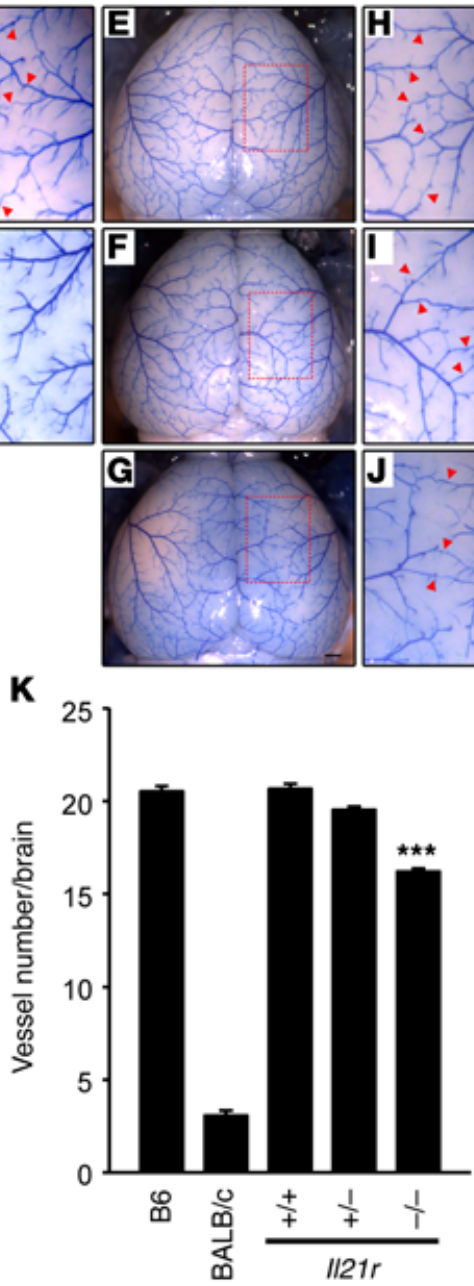

G

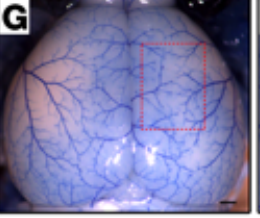

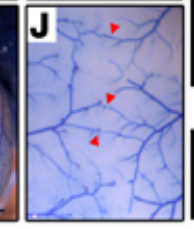
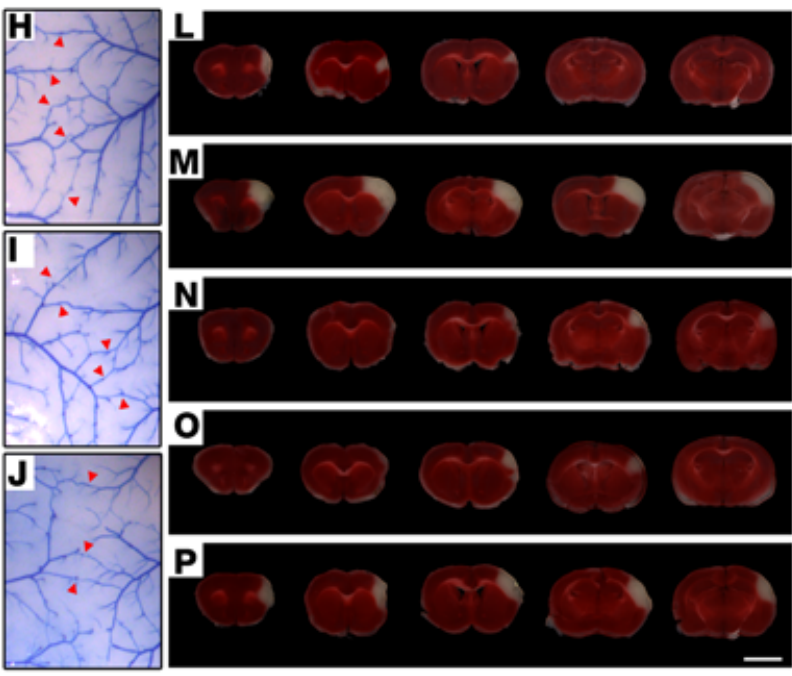

$\mathbf{Q}$

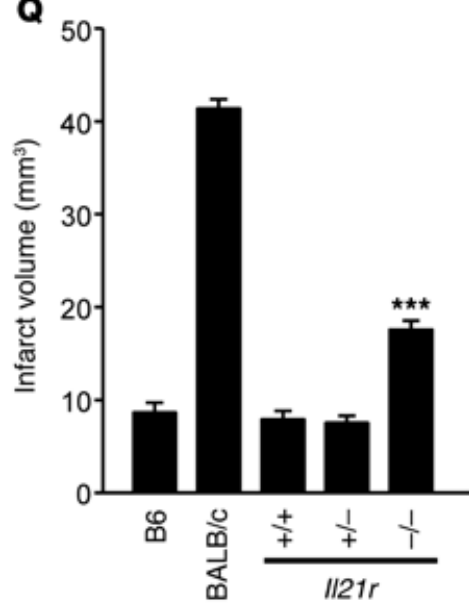

Figure 2. Collateral vessel anatomy and infarct volume after MCAO in II21r knockout mice. (A, B, and E-C) Representative images are shown for 2 inbred strains (B6, A; BALB/C, B) and II21r knockout mouse strains, WT (E), heterozygous (F), and KO (C). Scale bar: 1 mm. (C, D, and H-J) Photographs in C, D, H, I, and $\mathbf{J}$ are 3 times magnified from $\mathbf{A}, \mathbf{B}$, and $\mathbf{E}-\mathbf{G}$, respectively, and red arrowheads indicate vessel connections between ACA and MCA. (K) Graph indicates the average number of collateral vessel connections in the brain. Total number of animals for B6, BALB/c, II21r WT, II21r Het, and II21r KO are 33, 30, 40, 61,

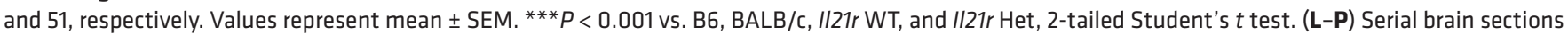
$(1 \mathrm{~mm})$ from B6 (L), BALB/C (M), and each genotype of II21r (WT, N; Het, 0; KO, P) 24 hours after MCAO. Infarct appears as white tissue after TTC staining. Scale bar: $5 \mathrm{~mm}$. (Q) Graph shows infarct volume for B6, BALB/c, II21r WT (+/+), II21r Het (+/-), and II21r KO. Total number of animals are 32, 30, 30, 36, and 47, respectively. Values represent mean \pm SEM. ${ }^{* *} P<0.001$ vs. B6, BALB/c, II21r WT, and II21r Het, 2-tailed Student's $t$ test.

mental Figure 1; supplemental material available online with this article; doi:10.1172/JCI84491DS1). Through the analysis of collateral vessel phenotypes, we found that Canq1 (collateral vessel number) also segregated in the $\mathrm{FVB} \times \mathrm{BALB} / \mathrm{c}$ and $\mathrm{NOD} \times \mathrm{BALB} / \mathrm{c}$ crosses. Importantly, the peak of these linkage signals for both Civq1 and Canq1 in all of these crosses is essentially identical. In contrast, although the $\mathrm{B} 6 \times \mathrm{A} / \mathrm{J}$ cross also showed highly significant (LOD score of 11.41) linkage to chromosome 7, upon more refined analysis obtained in a cross between a consomic line (CSS7: a B6 strain containing 2 copies of an A/J chromosome 7) and $\mathrm{B} 6$, we found that the peak of the linkage signal $(\mathrm{LOD}=16.06$; D7Mit130) is distinct (approximately $18 \mathrm{Mb}$ proximal) from the Civq1 and Canq1 loci. Since inbred strain A/J appears to segregate a different locus than that found in most of the other strains, we excluded haplotypes from $\mathrm{A} / \mathrm{J}$ from our shared haplotype analysis. We then identified ancestral SNP haplotype blocks that are shared among B6, FVB, and NOD (small infarct volumes and high collat- eral vessel density), but that are different from BALB/c and SWR (large infarct volumes and low collateral vessel density) (Figure 1A and Supplemental Table 1). The shared haplotype regions overlap with only 12 candidate genes for the major determinant or determinants of Civq1 and Canq1.

To identify the gene or genes underlying this locus, we first sought evidence of strain-specific transcript levels between B6 and BALB/c caused by regulatory sequence variation that might underlie the molecular basis of the QTL. We performed quantitative real-time PCR (qRT-PCR) for all 12 candidate genes in relevant tissues, including P1 and adult (12 week) brain cortex. As expected, the transcript 4933440M02Rik was not detected, since this gene is exclusively expressed in the testis (BioGPS 71208; http://biogps.org). Most of the other candidate genes exhibited slight but statistically significant expression differences, yet only 1 gene, $I l 21 r$, showed a more than 5 -fold expression difference between $\mathrm{B} 6$ and $\mathrm{BALB} / \mathrm{c}$ in $\mathrm{P} 1$ brain cortex and a 2 -fold higher 

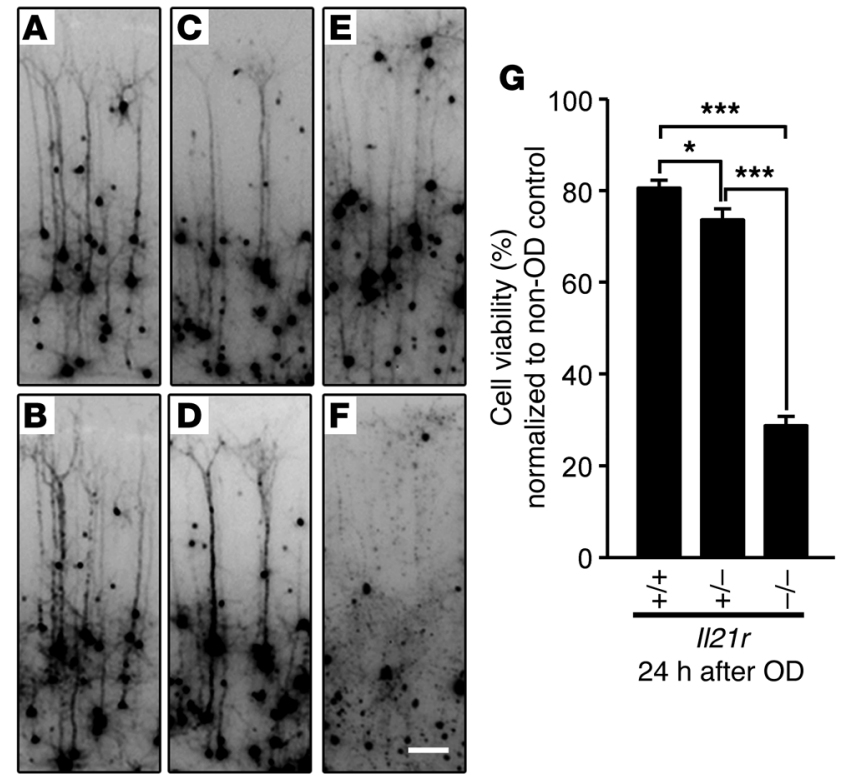

transcription level in $\mathrm{B} 6$ than in BALB/c adult brain cortex (Figure $1 \mathrm{~B}$ and Supplemental Figure 2). To further investigate this strain-specific transcription difference, we performed allele-specific gene expression analysis using F1 $(\mathrm{B} 6 \times \mathrm{BALB} / \mathrm{c})$ animals from relevant tissues that included $\mathrm{P} 1$ and adult brain cortex and embryonic macrophages (at E18.5), the latter due to their role as cellular chaperones in the development of vascular anastomoses and endothelial tip cell fusion (11). Similar to the qRT-PCR data, the B6 Il21r allele showed higher transcript levels than the BALB/C Il21r allele in 3 different tissues, embryonic macrophage (13.4fold), P1 (2.6-fold), and adult brain cortex (2.2-fold) (Figure 1C). We noted that the difference in expression between the $\mathrm{B} 6$ and $\mathrm{BALB} / \mathrm{c}$ alleles was especially dramatic in embryonic macrophages that play a role in vascular development.

Absence of Il21r modulates both cerebral collateral vessel anatomy and ischemic infarct volume. To further investigate whether Il21r modulates ischemic infarct volume, we examined relevant phenotypes of an $\mathrm{Il}^{2} \mathrm{r}^{--}$(KO) mouse strain, representing a complete loss-of-function allele of this candidate gene. We employed an Il21r KO mouse strain that has reportedly been maintained in the B6 strain background for many generations (12). Using wholegenome SNP genotyping, we confirmed that this KO allele is fully contained in the B6 background (data not shown), thereby eliminating unwanted genetic background effects. We first examined effects of the loss of this gene on pial collateral vessel anatomy. For each genotype of Il21r KO mice, we measured the number of vessel connections between the anterior cerebral artery (ACA) and middle cerebral artery (MCA) (Figure 2, E-J). The number of vessel connections in Il21r KO ( 16.2) was slightly reduced compared with both WT $\left(I l 21 r^{+/+}\right.$, referred to here as Il21r WT) ( 20.7) and heterozygous $\left(\mathrm{Il}_{21 r^{+/}}\right.$, referred to here as $\left.\mathrm{Il21} r \mathrm{Het}\right)$ ( 19.5) animals (Figure $2 \mathrm{~K})$. However, this difference is much more modest than the extreme difference seen between inbred strains B6 and BALB/C ( 20.5 vs. 3.1) (Figure 2, A-D, and K). We also measured cortical vessel connections between the MCA and the posterior cerebral artery (PCA) (Supplemental Figure 3). The number of collateral
Figure 3. II21r KO mice show increased neuronal cell death in the cortical brain slice ischemia assay. (A-F) Number of YFP-transfected cortical pyramidal neurons in mouse brain slices (II21r WT, II21r Het, and II21r KO) were compared before (A, C, and $\mathbf{E}$ ) and 24 hours after 30 minutes of OD (B, D, and $\mathbf{F}$ ). Photographs shown are magnified from a brain slice image shown in Supplemental Figure 7 (A-F). Scale bar: $0.2 \mathrm{~mm}$. (G) Graph indicates the average cell viability determined by observing the same neurons before and 24 hours after OD. II21r KO showed $70 \%$ increased neuronal cell death, while II21r WT and Het showed $20 \%$ and $26 \%$ increased neuronal cell death. Experiments were performed 3 times using 3 to 5 mice per genotype for each experiment. Total number of animals: WT, $n=10$; Het, $n=9$; KO, $n=13$. Number of brain slices analyzed for each genotype were 28,32 , and 35 for $1121 \mathrm{r}$ WT, $1 / 21 \mathrm{r}$ Het, and $1 / 21 \mathrm{r} \mathrm{KO}$, respectively. Values represent mean \pm SEM. ${ }^{*} P<0.05$; ${ }^{* *} P<0.001,1$-way ANOVA followed by Scheffe's test.

vessel connections between the MCA and PCA (Il21r WT, 20.1; Il21r Het, 19.8 ; and Il21r KO, 16.6) were similar in magnitude to those between the ACA and MCA. Since even complete loss of Il21r only modestly affects collateral vessel number, these data suggest that Il21r is not the major gene underlying Canq1, the locus on distal chromosome 7 controlling collateral vessel anatomy.

In order to determine whether loss of Il21r had any effect on cerebral circulation during reperfusion, we measured cerebral blood flow (CBF) during transient (as contrasted with permanent) MCAO (Supplemental Figure 4, A-C). In contrast with permanent $\mathrm{MCAO}$, transient MCAO allows vascular circulation (reperfusion) after 45 minutes of ischemia. CBF during transient MCAO was unaffected by loss of the gene. Likewise, infarct volume after transient MCAO was not significantly different between Il21r WT and Il21r KO mice (Supplemental Figure 4, D-F).

We next examined the effects of loss of Il21r on ischemic infarct volume in the permanent occlusion model. We performed MCAO and measured infarct volume for each genotype of Il21r $\mathrm{KO}$ mice (Figure 2, N-P). Infarct volume in Il21r KO mice (18.3 $\mathrm{mm}^{3}$ ) was $\sim 2.3$-fold larger than that observed in both Il21r WT $\left(7.9 \mathrm{~mm}^{3}\right)$ and $\mathrm{Il} 21 \mathrm{r}$ Het $\left(7.7 \mathrm{~mm}^{3}\right)$ mice (Figure $\left.2 \mathrm{Q}\right)$. As with the collateral vessel phenotypes, these differences in infarct volume were not as dramatic as those observed between inbred strains B6 and BALB/c (8.6 $\mathrm{mm}^{3}$ vs. $41.3 \mathrm{~mm}^{3} ; \sim 5$-fold) (Figure 2, L, M, and Q). Nonetheless, even with only a minor effect on collateral vessel number, the loss of Il21r clearly showed a robust and rather large effect on infarct volume after permanent MCAO. This suggests that, although Il21r exhibits some collateral-dependent effects on the infarct phenotype, loss of Il21r may also contribute to a collateral-independent pathway.

Absence of Il21r impairs neuronal protection in the brain after ischemic stroke induction. We hypothesized that the increased infarct volume seen in $\mathrm{Il21r} \mathrm{KO}$ mice might be in large part due to collateral-independent effects on neuroprotection. We first investigated whether IL-21R is normally expressed in brain tissue. Il21 and Il21r are thought to be mainly produced in immune cells (13); both are also reportedly expressed in neurons in human brain tissue (14). We attempted to identify the source of both Il21 and Il21r in mouse brain tissue. Both IL-21 and IL-21R were clearly expressed on neurons in the mouse brain (Supplemental Figure 5). The common $\gamma$ chain receptor and downstream effectors JAK2 and STAT3 are also expressed in neurons (15-17), illustrating that the major components of this signaling pathway are also present in neurons. 

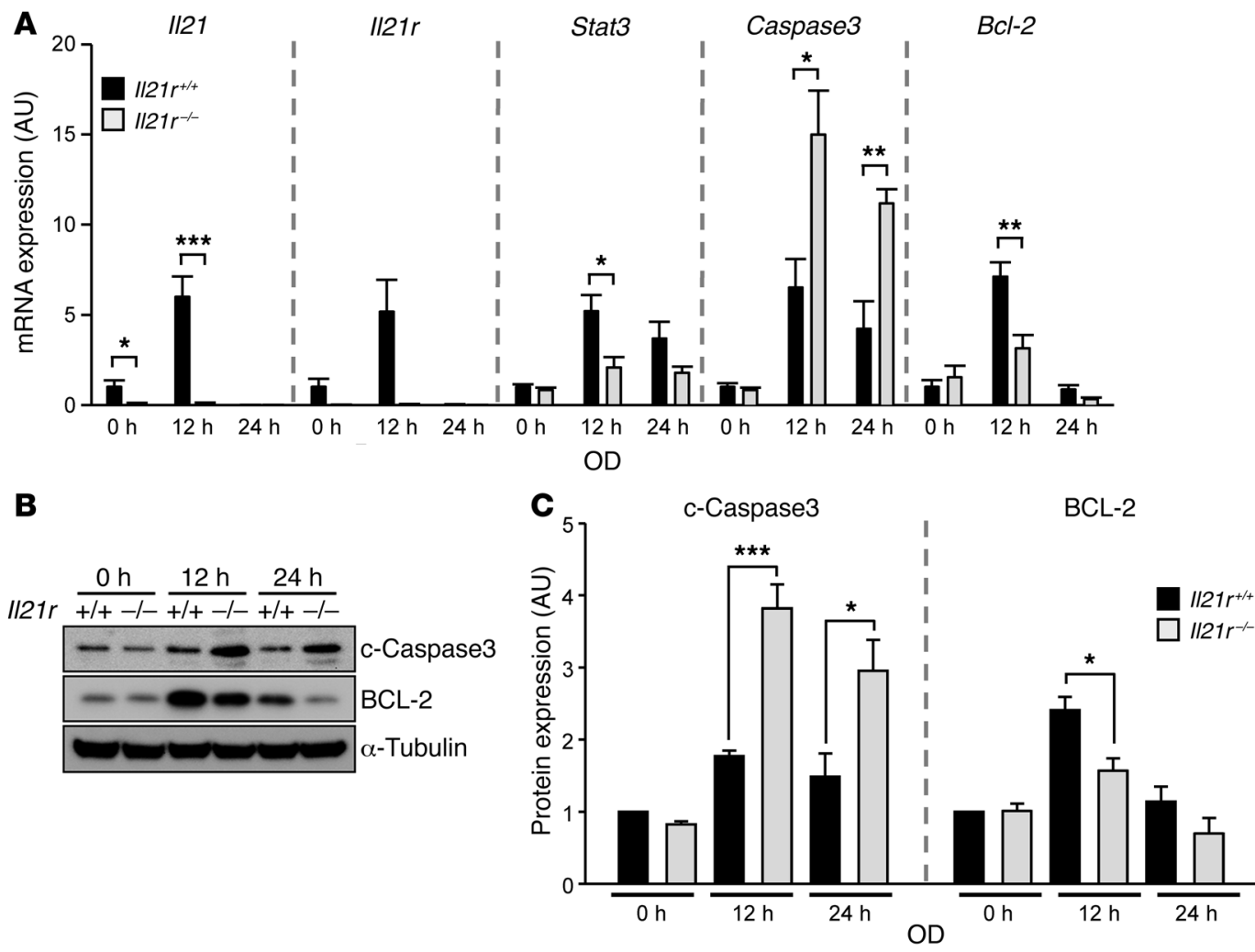

Figure 4. II21r KO increases cleaved caspase 3 after ischemic stroke stimulation in an ex vivo brain slice stroke model. (A) mRNA levels of II21, II21r, Stat3, caspase 3, and Bcl2 from II21r WT and II21r KO were determined by qRT-PCR 12 hours and 24 hours after 30 minutes of OD. Gapdh was used for normalization. Experiments were performed 3 times using 3 mice per genotype for each experiment. Values represent mean \pm SEM. (B) Western blots were performed to detect both cleaved caspase 3 (c-caspase 3) and BCL-2 in explanted brain slices of II21r WT (+/+) and II21r KO (-/-) 12 and 24 hours after 30 minutes of OD. (C) Levels of both cleaved caspase-3 and BCL-2 protein were determined. $\alpha$-Tubulin was used for normalization. Experiments were performed 4 times using 3 mice per genotype for each experiment. Values represent mean $\pm \mathrm{SEM}$. ${ }^{*} P<0.05$; ${ }^{*} P<0.01$; ${ }^{* *} P<0.001$, 2-tailed Student's $t$ test.

To determine whether Il21r modulates neuronal cell death through a collateral-independent mechanism, we employed an ex vivo study of intact brain slices under in vitro oxygen deprivation (OD). The ex vivo brain slice assay maintains the complex multicellular architecture of the tissue throughout the ischemic insult while in the absence of circulation, eliminating reperfusion effects of the collateral vessels. We were especially concerned to obtain accurate measures of neuronal cell death in this ex vivo model of stroke. Commonly, a brain slice is deprived of both oxygen and glucose (oxygen and glucose deprivation [OGD]). However, the OGD model requires that different brain slices are examined and averaged for the pre- and posttreatment arms of the experiment $(18,19)$, thereby introducing a potential source of variability and noise in the measurements. We included a modified procedure of $\mathrm{O}_{2}$ deprivation only, so as to assess cell viability with more reproducible and less variable results. Biolistic transformation of a yellow fluorescent protein (YFP) transgene primarily targets the neurons within the cultured brain slice (19). A predetermined number of cortical pyramidal neurons expressing YFP from each slice are chosen without bias, and these selected neurons from the brain slices are monitored to determine neuronal cell death after OD treatment. This assay is therefore internally controlled, and more accurate counts of neuronal cell death can be obtained.

We first validated the OD assay using brain slices obtained from inbred strains $\mathrm{B} 6$ and $\mathrm{BALB} / \mathrm{c}$, comparing cell viability for both the OGD and OD assays. Consistent with the trend seen in infarct volumes of B6 and BALB/c animals (Figure 2Q), BALB/c brain slices exhibit approximately $55 \%$ more neuronal cell death under OGD and approximately 52\% more neuronal cell death under OD than B6 brain slices (Supplemental Figure 6, A and B).

Having determined that OD is as effective as OGD in simulating ischemia in the ex vivo brain slice assay, we then examined neuronal cell death in $I l 21 r$ KO mouse strains. Figure 3 illustrates the advantages of the OD brain slice assay, as it enables the experimenter to follow the fate of individual neurons expressing YFP before and after OD treatment (Figure 3, A-F). Each individual neuron expressing YFP was magnified from each brain slice of Il21r KO strains (Supplemental Figure 7) for analysis. Using this assay, brain slices from $\mathrm{Il} 21 \mathrm{r} \mathrm{KO}$ mice showed an approximately $70 \%$ increase in neuronal cell death after OD treatment, compared with Il21r WT and Il21r Het brain slices, which showed approximately $20 \%$ and $26 \%$ neuronal cell death, respectively (Figure 3G). This is an approximately 3-fold increase in neuronal cell death due to the loss of Il21r. Importantly, these effects of Il21r are independent of tissue reperfusion through the collateral vessels and represent an innate neuroprotective effect.

IL-21R signals through the JAK/STAT pathway in brain slices and affects apoptosis. IL-21R is one of the class I cytokine receptors, which include IL-2R, IL-4R, IL-7R, IL-9R, IL-15R, and IL-21R (13). $\mathrm{IL}-21 \mathrm{R}$ forms a heterodimeric receptor complex with the common 
A

\begin{tabular}{|c|c|c|c|c|c|c|c|c|c|c|c|c|c|c|c|c|c|c|c|c|c|}
\hline & 190 & & & & & & & & & & 200 & & & & & & & & & & 210 \\
\hline C57BL/6J, FVB/NJ, NOD/ShiLtJ & $\mathrm{F}$ & $\mathrm{H}$ & $\mathrm{K}$ & D & $\mathrm{s}$ & $\mathrm{s}$ & $\mathrm{Y}$ & Q & $\mathrm{L}$ & Q & V & $\mathrm{R}$ & A & A & $\mathrm{P}$ & Q & $P$ & G & $\mathrm{T}$ & $\mathrm{s}$ & $\mathrm{F}$ \\
\hline BALB/c, SWR/J & $\mathrm{F}$ & $\mathrm{H}$ & $\mathrm{K}$ & D & $\mathrm{s}$ & $\mathrm{s}$ & $\mathrm{Y}$ & Q & L & Q & M & $\mathrm{R}$ & A & A & $\mathrm{P}$ & Q & $P$ & G & $\mathrm{T}$ & $\mathrm{s}$ & $\mathrm{F}$ \\
\hline Rat & $\mathrm{F}$ & Q & K & D & $S$ & S & $Y$ & Q & L & Q & v & $\mathrm{R}$ & A & A & $P$ & Q & $P$ & G & $\mathrm{T}$ & S & $F$ \\
\hline Chinese hamster & $\mathrm{F}$ & $\mathrm{H}$ & K & G & $S$ & $\mathrm{~T}$ & $Y$ & Q & L & Q & v & $\mathrm{R}$ & A & A & $P$ & Q & $\mathrm{T}$ & $S$ & S & S & $F$ \\
\hline Guinea pig & $\mathrm{F}$ & $R$ & K & $\mathrm{N}$ & S & S & Y & $E$ & L & Q & v & $\mathrm{R}$ & A & G & $P$ & Q & $P$ & G & S & A & $F$ \\
\hline Naked mole rat & $\mathrm{F}$ & $R$ & K & N & $S$ & S & Y & $\mathrm{E}$ & L & Q & V & $R$ & A & G & $P$ & Q & $P$ & G & S & $\mathrm{F}$ & $F$ \\
\hline Little brown bat & $\mathrm{F}$ & $\mathrm{H}$ & $P$ & G & A & S & $Y$ & $E$ & L & Q & V & $\mathrm{R}$ & A & G & $P$ & Q & $P$ & G & S & S & $F$ \\
\hline Human & $\mathrm{F}$ & $\mathrm{R}$ & K & D & $S$ & $S$ & $Y$ & $\mathrm{E}$ & L & Q & V & $\mathrm{R}$ & A & G & $P$ & M & $P$ & G & $S$ & S & $Y$ \\
\hline Horse & $\mathrm{F}$ & $\mathrm{R}$ & G & D & $S$ & $S$ & $Y$ & E & L & $Q$ & V & $\mathrm{R}$ & A & G & $P$ & $Q$ & $P$ & G & $S$ & S & $F$ \\
\hline Bovine & $\mathrm{F}$ & $\mathrm{H}$ & $S$ & G & $S$ & $S$ & $Y$ & $E$ & L & Q & v & $R$ & A & G & $\mathrm{P}$ & $Q$ & $P$ & G & S & $\mathrm{T}$ & $\mathrm{F}$ \\
\hline Sheep & $\mathrm{F}$ & $\mathrm{H}$ & $S$ & G & $S$ & $\mathrm{~N}$ & $Y$ & $\mathrm{E}$ & L & Q & v & $\mathrm{R}$ & A & G & $P$ & $Q$ & $P$ & G & $S$ & $\mathrm{~T}$ & $F$ \\
\hline African elephant & $\mathrm{F}$ & $\mathrm{H}$ & K & G & $S$ & $S$ & $Y$ & E & V & Q & v & $R$ & A & G & $P$ & Q & $P$ & G & $S$ & S & $\mathrm{F}$ \\
\hline Chimpanzee & $\mathrm{F}$ & $\mathrm{R}$ & K & $\mathrm{D}$ & $S$ & $s$ & $Y$ & E & L & Q & v & $\mathrm{R}$ & A & A & $P$ & M & $P$ & G & $S$ & S & Y \\
\hline umatran orangutan & $\mathrm{F}$ & $R$ & K & $\mathrm{D}$ & $S$ & $S$ & Y & $\mathrm{E}$ & L & Q & V & $R$ & A & G & $P$ & M & $P$ & G & $S$ & S & $Y$ \\
\hline thern white-cheeked gi & $\mathrm{F}$ & $R$ & K & D & $S$ & $S$ & $Y$ & E & L & Q & V & $R$ & A & G & $P$ & M & $P$ & G & S & S & \\
\hline Rhesus macaque & $\mathrm{F}$ & $R$ & K & D & S & $S$ & Y & $E$ & L & Q & $\mathrm{v}$ & $\mathrm{R}$ & A & G & $\mathrm{P}$ & M & $P$ & G & S & S & \\
\hline
\end{tabular}

$\mathbf{B}$

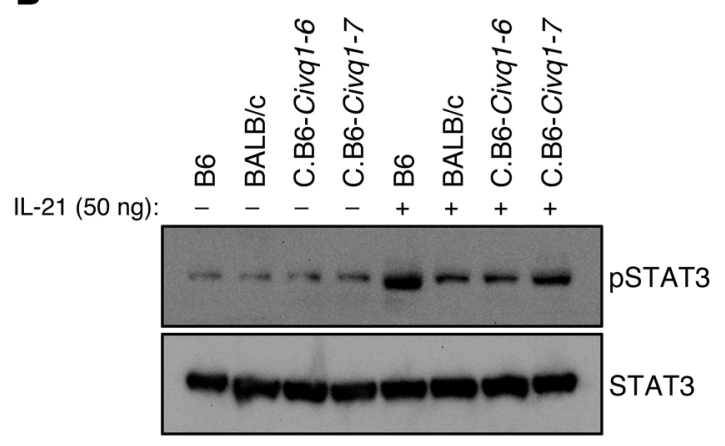

C

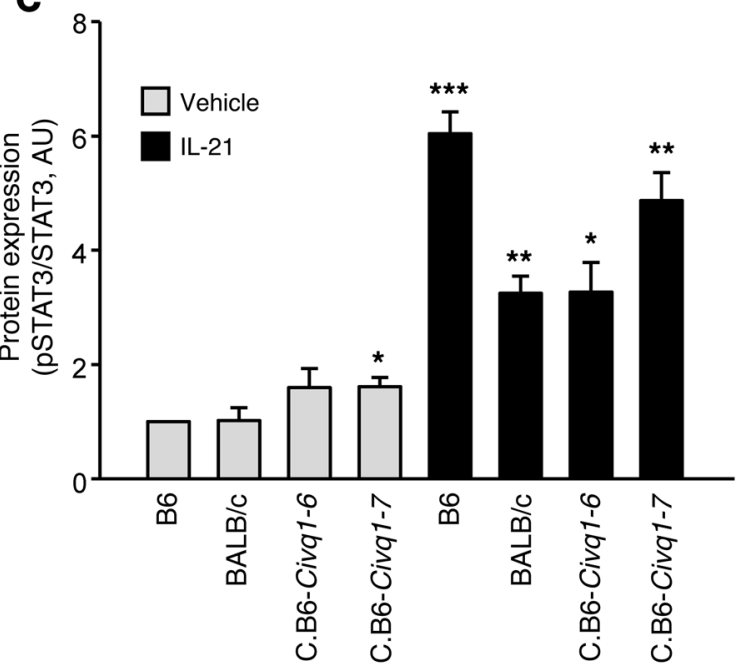

D

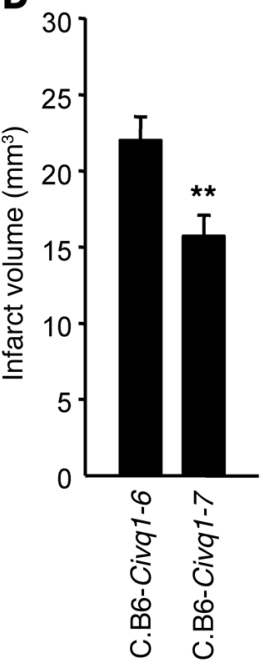

Figure 5. Functional consequences of IL-21R are altered by a second IL-21R coding difference between inbred mouse strains. (A) Alignments of amino acid sequences are compared across different inbred mouse strains and other mammalian species. The valine (V) found at amino acid position 200 (rs33006504) in B6, FVB, and NOD mouse strains is well conserved in mammalian species except inbred mouse strains BALB/c and SWR showing the methionine (M) at the amino acid position. This V200M change is predicted to be functionally damaging to the protein with in silico analysis (PolyPhen-2 score: 0.789). (B) Western blots were performed to detect levels of both p-STAT3 and STAT3 in explanted brain slices of 2 inbred mouse strains, B6 and BALB/C, as well as 2 Civq1 congenic lines, C.B6-Civq1-6 and C.B6-Civq1-7, 1 hour after treatment with either vehicle or recombinant murine IL-21 (50 ng/ $\mathrm{ml}$ ). (C) Levels of p-STAT3 were determined. Level of total STAT3 was used for normalization. Experiments were performed 3 times using 3 mice per mouse strain for each experiment. Values represent mean \pm SEM. ${ }^{*} P<0.05 ;{ }^{* *} P<0.01$; ${ }^{* *} P<0.001$ vs. B6 vehicle treatment, 2 -tailed Student's $t$ test. (D) Graph shows the infarct volume. Total numbers of animals for C.B6-Civq1-6 and C.B6-Civq1-7 are 29 and 30, respectively. Representative images are shown in Supplemental Figure 13, F and G. Values represent mean \pm SEM. ${ }^{* *} P<0.01$ vs. C.B6-Civq1-6, 2-tailed Student's $t$ test.

cytokine receptor $\gamma$ chain $\left(\gamma_{c}\right)$ to transmit signals through the JAK/ STAT pathway. Although IL-21/IL-21R activates STAT1, STAT3, STAT5A, and STAT5B, STAT3 is the predominant effector (13). Activated STAT3 is involved in a wide range of biological functions (20-22). Interestingly, mice with cardiomyocyte-restricted deletion of Stat3 exhibit enhanced susceptibility to myocardial ischemia/reperfusion injury and infarction, with increased cardiac apoptosis and reduced cardiac function and survival (20). Additionally, a recent study demonstrated that the absence of Il21r impaired perfusion recovery after hindlimb ischemia (HLI) (23). These data showed that in HLI, Il21r is upregulated, which increases the ratio of BCL-2/BAX through IL-21/IL-21R/STAT3 signaling, leading to reduced apoptosis and increased cell survival (23). We wanted to determine whether Il21r also affected apoptosis in cerebral ischemia, focusing on BCL-2 and cleaved caspase 3, antiapoptotic and apoptotic factors, respectively, that are regulated through STAT3 phosphorylation (p-STAT3). We found that mRNA transcription levels of Stat3 and Bcl2 in Il21r KO mouse brain slices showed significant downregulation 12 hours after transient OD treatment compared with their mRNA transcription levels in Il21r WT mouse brain slices, whereas caspase 3 mRNA transcription levels in Il21r KO mouse brain slices were upregulated (Figure 4A). While mRNA transcription levels of Il21, Il21r, Stat3, and Bcl2 decreased 24 hours after transient OD treatment, caspase 3 mRNA transcription levels remained elevated (Figure 4A). The levels of both $I l 21$ and Il21r transcription in Il21r WT brain slices increased 12 hours after transient OD treatment compared with non-OD treatment (Figure 4A), but were undetectable 24 hours after transient OD treatment. Consistent with the mRNA transcription results, Il21r KO mouse 


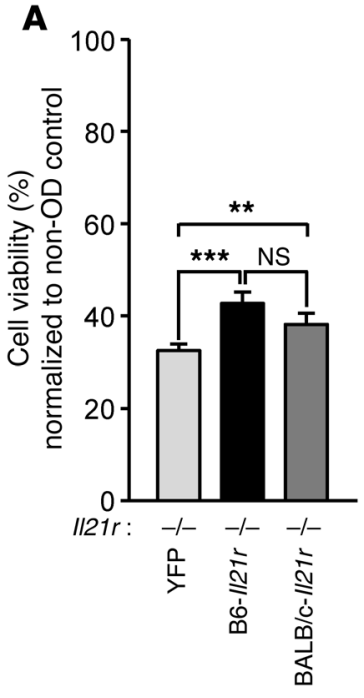

B

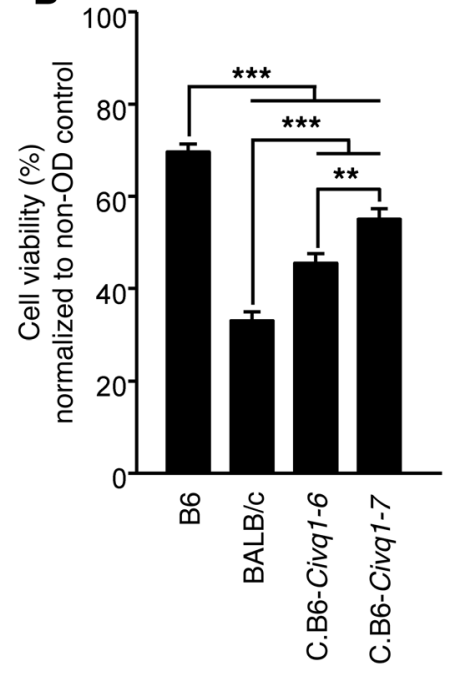

Figure 6. Neuronal cell death induced by ischemic stroke stimulation in explanted brain slices is rescued by the II21r transcript containing the B6 allele. (A) The II21r transcript was isolated and cloned from B6 and $\mathrm{BALB} / \mathrm{c}$ brain cortex, and each CDNA was introduced in gWIZ vector (Genlantis) containing YFP. Either empty, I/21r-B6, or I/21r-BALB/c containing YFP was introduced in explanted $I / 21 \mathrm{r} K \mathrm{~K}$ brain slices that are allowed to express YFP for 24 hours. Graph indicates average cell viability determined by following the same neurons before and 24 hours after OD. II21r KO explanted brain slices were transfected with I/21r cDNA from either B6 or $B A L B / c$, and neuronal cell viability was compared with transfection with empty (YFP alone) vector. Experiments were performed 3 times using 3 to 5 mice (II21r KO) for each experiment. Total number of animals: II21r KO, $n=13$. The numbers of brain slices analyzed for empty vector, II21r-B6, and $1121 r-B A L B / c$ were 96,88 , and 87 , respectively. Values represent mean \pm SEM. ${ }^{* *} P<0.01$; ${ }^{* *} P<0.001$, 1-way ANOVA followed by Scheffe's test. (B) Neuronal cell viability after $\mathrm{OD}$ for explanted brain slices was measured for 2 inbred mouse strains, B6 and BALB/c, as well as 2 Civq1 congenic lines, C.B6-Civq1-6 and C.B6-Civq1-7. Graph indicates average cell viability determined by observing the same neurons before and 24 hours after $\mathrm{OD}$. Experiments were performed 3 times using 3 to 5 mice per strain for each experiment. Total number of animals: B6, $n=9$; BALB/c, $n=9$; C.B6-Civq1-6, $n=12$; C.B6-Civq1-7, $n=13$. Numbers of brain slices analyzed for B6, BALB/c, C.B6-Civq1-6, and C.B6-Civq1-7 were 45, 39, 42, and 45 , respectively. Values represent mean \pm SEM. ${ }^{* *} P<0.01$; ${ }^{* *} P<$ $0.001,1$-way ANOVA followed by Scheffe's test.

brain slices showed significantly increased cleaved caspase 3 levels compared with $I l 21 r$ WT mouse brain slices after transient OD treatment (Figure 4, B and C), and as expected, BCL-2 levels in $I l 21 r \mathrm{KO}$ mouse brain slices were reduced after transient OD treatment (Figure 4, B and C). To determine whether the gene/ protein expression differences observed in the ex vivo brain slice system reflect the in vivo conditions, we determined the levels of cleaved caspase 3 and BCL-2 in the ipsilateral (the side where the MCA was occluded) hemisphere of the brain after permanent MCAO between $I l 21 r$ WT and KO animals (Supplemental Figure $8 \mathrm{~A})$. The levels of cleaved caspase 3 were slightly elevated in $I l 21 r$ KO compared with $I l 21 r$ WT, but the difference did not reach statistical significance. However, the levels of BCL-2 were significantly reduced in Il21r KO mice (Supplemental Figure 8B). We note that these in vivo and ex vivo stroke models differ in terms of effected area (a focal effect for the in vivo surgery mouse models vs. a global effect for the ex vivo brain slice ischemia models).
Nevertheless, the similar results observed for cleaved caspase 3 and BCL-2 expression across these 2 different models of ischemia show that loss of $I l 21 r$ induces apoptosis in ischemic stroke, suggesting a critical role for Il21r in ischemic neuronal protection.

SNP in Il21r modulates response to ischemic stroke damage. Il21r was chosen as a potential candidate gene for Civq1 due to its ancestral haplotypes that segregated with the infarct volume phenotype in the mapping strains as well as its differential gene expression in the mapping strains. We also noted that 2 nonsynonymous coding SNPs in IL-21R (Arg69Lys and Val200Met) segregated with infarct volume in these same strains with B6 (and some other small infarct volume strains) exhibiting both the Arg69 and Val200 variants and BALB/c (and other large infarct volume strains) showing the Lys69 and Met200 variants. Although both the Arg69 and the Val200 are well conserved among mammalian species, the Arg69Lys coding change was predicted to be functionally "benign" (Supplemental Figure 9). In contrast, in silico functional analysis using PolyPhen-2 (http:// genetics.bwh.harvard.edu/pph2/) predicted the methionine at position 200 to be functionally "damaging" (Figure 5A). This Val200Met polymorphism is located in the second fibronectin domain of IL-21R adjacent to the class I cytokine receptor signature motif (WSXWS, amino acid position 214-218) $(24,25)$. This motif is involved in proper protein folding, receptor internalization and transport to the plasma membrane, ligand binding, and signal transduction (25-27). Based on the crystal structure of the extracellular domain of IL-21R that complexes with IL-21 (Research Collaboratory for Structural Bioinformatics Protein Data Bank [RCSB PDB]: 3TGX) (28), we predicted slight structural differences between IL-21 $\mathrm{R}^{\mathrm{Val200}}$ and IL-21R $\mathrm{R}^{\mathrm{Me} 200}$ that might affect the function of the protein (Supplemental Figure 10). Using a protein-modeling program (PyMOL, https://www.pymol.org), we found that in the IL-21R $\mathrm{R}^{\mathrm{Val200}}$ variant, a $\beta$ strand containing Trp214 and an anti-parallel $\beta$ strand containing Val200 constituted the hydrophobic core. However, in the IL-21R ${ }^{\mathrm{Met200}}$ variant, the methionine side chain created steric hindrance between these $\beta$ strands. This steric hindrance may also interfered with C-mannosylation, where a mannose is attached to the first tryptophan (W) in the WSXWS motif. This physical difference could lead to altered levels of signaling between the 2 allelic variants of IL-21R.

To investigate whether these coding differences between $\mathrm{B} 6$ $\left(\mathrm{IL}-21 \mathrm{R}^{\mathrm{Val200}}\right)$ and BALB/c $\left(\mathrm{IL}-21 \mathrm{R}^{\mathrm{Met200}}\right)$ might play a role in the differential response to ischemic stroke brain damage, we measured IL-21R $\mathrm{R}^{\mathrm{Val} 200}$ and IL-21R $\mathrm{R}^{\mathrm{Met200}}$ responsiveness by observing levels of the downstream effector p-STAT3 after recombinant IL-21 treatment of brain slices from B6, BALB/c, and 2 Civq1 congenic lines. Civq1 congenic line C.B6-Civq1-7 carries a segment of B6 chromosome $7(\sim 1.9 \mathrm{Mb})$ introgressed into the BALB/c background and contains the B6 allele of Il21r. Civq1 congenic line C.B6-Civq1-6 also carries a segment of B6 chromosome 7 ( 5.1 $\mathrm{Mb}$ ) introgressed into the BALB/c background, but C.B6-Civq1-6 contains the BALB/c allele of Il21r (Figure 1A). We detected different levels of IL-21R responsiveness to recombinant IL-21 treatment between $\mathrm{B} 6$ and BALB/c brain slices, and also between brains slices of C.B6-Civq1-6 and C.B6-Civq1-7 (Figure 5, B and C). Brain slices from both B6 and C.B6-Civq1-7 showed dramatically increased p-STAT3 after treatment with recombinant IL-21 
as compared with brain slices from BALB/c and C.B6-Civq1-6 treated with recombinant IL-21 (Figure 5, B and C). This suggests that the IL-21R $\mathrm{R}^{\mathrm{Val} 200 \mathrm{Met}}$ polymorphism alters downstream signaling through p-STAT3.

To determine whether the different $\mathrm{p}$-STAT3 levels were due to alterations in the IL-21/IL-21R pathway (Figure 5, B and C, and Supplemental Figure 11, A and B), we determined p-STAT3 levels after treatment with recombinant ciliary neurotrophic factor (CNTF), which also signals through p-STAT3, but via the tripartite complex of CNTFR, leukemia inhibitory factor receptor (LIFR), and gp130 (29-32). p-STAT3 levels were rapidly increased 15 minutes after recombinant CNTF treatment in B6 brain slices (Supplemental Figure 11, C and D). Unlike the p-STAT3 levels observed after IL-21 treatment, there were no substantial differences in levels of p-STAT3 in brain slices of B6, BALB/c, C.B6-Civq1-6, and C.B6-Civq1-7 upon activation by CNTF signaling (Supplemental Figure 12, A and B). These data further support that the differential levels of p-STAT3 after IL-21 treatment among the strains result from the genetic variation of $I l 21 r$ - either at the level of expression or due to coding changes in the receptor or both.

To evaluate whether infarct volume was modulated by this natural genetic variation of $I l 21 r$, we performed MCAO to induce ischemic brain stroke using 2 inbred mouse strains and 2 Civq1 congenic lines. The infarct volumes in B6 $\left(8.6 \mathrm{~mm}^{3}\right)$, C.B6Civq1-6 (22.0 $\left.\mathrm{mm}^{3}\right)$, and C.B6-Civq1-7 $\left(15.7 \mathrm{~mm}^{3}\right)$ were dramatically reduced compared with the infarct volume in BALB/c (41.3 $\mathrm{mm}^{3}$ ). Importantly, the infarct volume in C.B6-Civq1-7, segregating the B6 allele of $I l 21 r$, was significantly reduced compared with the infarct volume in C.B6-Civq1-6, with the BALB/c Il21r allele (Figure 2Q, Figure 5D, and Supplemental Figure 13, F and G).

Since collateral vessel number is also a major determinant of infarct volume, we wanted to determine whether the Val200Met polymorphism in IL-21R affected collateral vessel number. We noted that the vessel connections in both C.B6-Civq1-6 ( 13.7) and C.B6-Civq1-7 ( 17.5) were highly increased in number compared with the vessel connections in BALB/c ( 3.1) (Figure 2K and Supplemental Figure 13, A-E). The congenic lines, however, did not differ significantly in vessel numbers from those of B6, indicating that the Val200Met polymorphism in IL-21R does not seem to have a robust effect on collateral vessel number. In neither of the congenic lines did the infarct volumes or the collateral vessel numbers equal the values seen in the mapping strains. Natural genetic variation of Il21r affected infarct volume and, to a lesser extent, collateral vessel number, but the effects on collateral vessels were modest, suggesting the involvement of another gene or genes in the locus on chromosome 7. This mirrors the results for collateral vessel number and infarct volume in Il21r $\mathrm{KO}$ mice (Figure 2), demonstrating that loss of Il21r modulates ischemic brain stroke primarily in a collateral-independent manner.

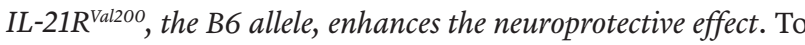
investigate whether the IL-21/IL-21R pathway determines cell viability after ischemic stroke, we performed an ex vivo brain slice assay using Il21r KO mouse brain slices transfected (rescued) with either B6-Il21r, BALB/c-Il21r, or empty vector containing YFP. After transient OD treatment, brain slices expressing B6-Il21r and BALB/c-Il21r showed significantly increased neuronal cell viability compared with YFP control brain slices $(\sim 3 \%$ and $~ 39 \%$, respectively, vs. 30\%) (Figure 6A). However, the inefficiency of transfection and its variable effects limits our ability to determine the full effects of the transfected gene on ischemia-induced cell viability. Thus, we also directly measured cell viability of the 2 Civq1 congenic lines that harbor either the B6 or BALB/c Il21r variant and compared cell viability with that in their parental inbred strains. The 2 inbred mouse strains, B6 and BALB/c, showed dramatically different cell viability rates $(70 \%$ and $\sim 30 \%$, respectively) after transient OD treatment as compared with before OD treatment of their brain slices (Figure 6B). Both C.B6-Civq1-6 and C.B6-Civq1-7 clearly showed increased cell viability $(\sim 45 \%$ and $\sim 55 \%$, respectively) after transient OD treatment compared with before OD treatment. Importantly, the differences between the congenic lines containing either the $\mathrm{B} 6$ or BALB/c allele at $I l 21 r$ (B6: C.B6-Civq1-7 and BALB/c: C.B6-Civq1-6) also are statistically significant (Figure 6B). Although cell viability does not reach the levels of the parental strains, the genotype at $I l 21 r$ in congenic lines mirrors the trends of the parental strains. We note that the locus on chromosome 7 (Civq1) may harbor another gene or genes modulating infarct volume. Our previous finding that Itgal modulates infarct volume in Civq1 (3) may explain in part why C.B6Civq1-6, containing the BALB/c allele of $I l 21 r$, showed increased neuronal cell viability compared with the mapping strain, BALB/c. Nonetheless, these data show that the B6 allele of $I l 21 r$ increases neuronal cell viability and thus leads to decreased infarct volume.

\section{Discussion}

Although a number of studies have identified environmental and genetic risk factors for ischemic stroke, the natural genetic risk factors that modulate neural tissue damage (an infarct) in the brain have been difficult to identify. Our understanding of the mechanisms underlying infarct damage is based primarily on loss- or gain-of-function experiments in genetically engineered animal models. However, evidence based on gene ablation or gene overexpression in transgenic mice does not always provide insight into mechanisms involved in the natural disease state. Experimental gene deletion or transgenic overexpression creates an artificial genetic and physiological state often far removed from that found in naturally occurring disease. Therefore, we have attempted to understand the biological processes involved in infarction by exploiting naturally occurring, inbred strainspecific phenotypic differences in cerebral infarct volume. Using multiple F2 crosses, we have previously fine-mapped a locus, Civq1 $(3,4)$, on chromosome 7 that overlaps with a locus controlling collateral vessel number, Canq1 (7). We generated additional F2 crosses $(\mathrm{B} 6 \times \mathrm{A} / \mathrm{J}, \mathrm{FVB} \times \mathrm{BALB} / \mathrm{c}$, and $\mathrm{NOD} \times \mathrm{BALB} / \mathrm{c})$ for Canq1 and validated that collateral vessel traits shared the same region of Civq1. Approximately 124 known genes are found within the 1.5 LOD interval of this locus on chromosome 7. To further reduce the number of candidate genes, we employed interval-specific SNP haplotype analysis using the 5 inbred mouse strains that harbor this locus in mapping crosses. Through this analysis, we identified 12 candidate genes for further consideration. One of these genes, Il21r, shows the largest strain-specific differences in transcript levels between 2 inbred strains, B6 and $\mathrm{BALB} / \mathrm{c}$, and also harbors a coding SNP predicted by modeling to alter receptor function. 
IL-21R is a type I cytokine receptor that, when activated by its cytokine (IL-21), signals through the JAK/STAT pathway to modulate innate and adaptive immunity (33) as well as cell survival (3436). Il21r maps within a genetic locus determining limb necrosis and recovery of perfusion ( $L s q 1)$ that shares the same location as Civq1 on chromosome 7 (37). Il21r was recently shown to enhance perfusion recovery in an HLI model by preventing apoptosis (23). With the physiological similarities between ischemic models and the shared map position of the loci, Il21r is a compelling candidate gene as a modulator of infarction in cerebral ischemia.

We examined both collateral vessel density and infarct volume after MCAO in the Il21r KO mouse. Although infarct volumes are more than 2.3-fold larger in Il21r KO mice compared with $I l 21 r$ WT and Il21r Het mice, there were only slightly fewer collateral vessel connections in the Il21r KO mice. These data suggest that, at least in part, Il21r modulates infarct volume in cerebral ischemia through a collateral-independent (nonvascular) mechanism. A nonvascular neuroprotective role was validated using an ex vivo brain slice OD assay that eliminates effects of vascular circulation. Under OD, Il21r KO brain slices showed increased neuronal cell death and exhibited increased levels of the proapoptotic factor cleaved caspase 3 and decreased levels of the antiapoptotic factor BCL-2. These combined data further support a neuroprotective role for Il21r in cerebral ischemia.

A nonsynonymous coding SNP in Il21r may also play a role in the different cerebral infarct volumes observed between the inbred mouse strains B6 and BALB/c. This SNP is located near the WSXWS motif (amino acid position 214 218) in the second fibronectin domain of IL-21R extracellular portion, a region of the protein thought to be involved in ligand binding and signaling transduction. The BALB/c allele IL-21R $\mathrm{R}^{\mathrm{Met} 200}$ is predicted to alter the structure of this motif in IL-21R, and our results showed that signal transduction as measured by $\mathrm{p}$-STAT3 levels was reduced compared with signaling by the B6 IL-21R $\mathrm{R}^{\mathrm{Val} 200}$ allele. These signaling transduction differences caused by the naturally occurring SNP in Il21r also segregate with neuronal cell death and infarct volume in the mouse model of cerebral ischemia.

A mutation in the human Il21r gene (Arg201Leu) causes a primary immunodeficiency syndrome (38). The mutant IL-21R ${ }^{\text {Leu201 }}$ receptor is retained in the ER and nuclear membrane, indicating aberrant receptor trafficking. This abnormal subcellular distribution of IL-21R leads to impaired signal transduction (38). We did not observe any differences in cellular localization between B6-

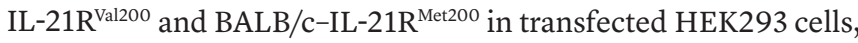
yet we did find slightly increased p-STAT3 levels in cells expressing IL-21R $\mathrm{R}^{\mathrm{Val200}}$ versus IL-21R $\mathrm{R}^{\mathrm{Met} 200}$ upon IL-21 stimulation (data not shown). Since the IL-21R $\mathrm{R}^{\text {Arg201Leu }}$ human variant is a rare, disease-causing mutation, the consequences of this change might be expected to be more severe than those of the IL-21R ${ }^{\text {Val200Met murine }}$ variant that is present as a common SNP in the mouse genome.

Our schematic model (Supplemental Figure 14) postulates that under ischemic stroke, the B6 allele of IL-21R shows enhanced signal transduction, whereas the BALB/c allele shows impaired signal transduction. These signaling differences are most likely due to a combination of strain-specific differences in the levels of the IL-21R receptor as well as a critical coding polymorphism in the protein. These signal transduction differences differentially induce p-STAT3 levels in B6 and BALB/c mice that in turn alter levels of both antiapoptotic (e.g., BCL-2) and proapoptotic (e.g., cleaved caspase 3) factors. Furthermore, p-STAT3 has been shown to act as a transcription factor determining IL-21R levels $(23,35$, $36,39,40)$, suggesting that this pathway acts as a positive feedback loop under cerebral ischemia.

In this study, we demonstrate for what we believe is the first time that $I l 21 r$ is directly involved in modulating infarct volume in cerebral ischemia via neuroprotection. While these effects were striking and consistent in the permanent MCAO model, such dependence on Il21r was curiously not evident in the transient MCAO model. This may be due to the largely different pathophysiologies that are known to dominate in the transient, ischemia-reperfusion vs. permanent MCAO models of cerebral ischemia (41). In fact, after transient MCAO, Il21 KO mice have been reported to exhibit smaller rather than larger infarct volumes as well as improved neurological function (42). However, in this case, the source of IL-21 in the transient MCAO model was shown to be predominantly from infiltrating $\mathrm{T}$ lymphocytes associated with reperfusion injury. In contrast, T lymphocyte infiltration was not observed in the permanent MCAO model. The dependencies we report here on $I l 21 r$ expression and allelic variants are thus more likely to be mediated through resident IL-21 and IL-21R, which are known to be expressed primarily in neurons under conditions of permanent vessel occlusion (14).

Cerebral infarction has been postulated to be the result of a synergistic imbalance in the neurovascular unit consisting of neurons, astrocytes, macrophages, and vascular structures (43). Our genetic data suggest that a number of different genetic loci can modulate the size of the infarct upon cerebral ischemia $(4,5,44$, 45). Future studies investigating other candidate genes at this and other loci will identify additional genes that are critical to this synergistic neurovascular regulation.

\section{Methods}

Animals. All inbred mouse strains were obtained from the Jackson Laboratory or bred locally from breeding pairs of each strain. The $I l 21 \mathrm{r}^{\circ-}(\mathrm{KO})$ mice in the B6 mouse background were provided by Warren J. Leonard of the National Heart, Lung, and Blood Institute (Bethesda, Maryland, USA), who originally generated these mice. Mice (male and female animals) were age matched (P10 for brain slice culture, P21 for collateral vessel perfusion, and $12 \pm 1$ week for MCAO) for all experiments.

Interval-specific SNP haplotype analysis. For the 9.6-Mb interval on chromosome 7, SNP data were obtained from the Mouse Phenome Database (http://phenome.jax.org/), the Wellcome Trust Sanger Institute Mouse Genomes Project (http://www.sanger.ac.uk/sanger/ Mouse_SnpViewer/rel-1505), and the Center for Genome Dynamics (http://cgd.jax.org). Physical map position was based on the genomic sequence from the GRCm $38 / \mathrm{mm} 10$. Haplotype blocks were defined as 3 or more adjacent informative SNPs shared between the large infarct strains $(\mathrm{BALB} / \mathrm{c}$ and $\mathrm{SWR} / \mathrm{J})$ that differed from the haplotype for the small infarct strains (B6, FVB, and NOD).

Isolation of embryonic macrophages. E18.5 embryonic brain was diced into small pieces, incubated in DMEM containing 10\% FBS and collagenase type IV $(0.2 \mathrm{mg} / \mathrm{ml}$, Sigma-Aldrich) for 30 minutes at $37^{\circ} \mathrm{C}$, and then passed through a 19 -gauge syringe to obtain a homogeneous cell suspension. Cells were washed with PBS supplemented 
with $0.5 \mathrm{mM}$ EDTA and 0.5\% BSA and incubated with $10 \mu \mathrm{l}$ antiCD11b (macrophage) antibody-conjugated magnetic beads (Miltenyi Biotech, catalog 130-049-601) for 15 minutes on ice. These cells were applied to MACS MS separation columns on magnetic stands and washed with $1.5 \mathrm{ml}$ PBS supplemented with $0.5 \mathrm{mM}$ EDTA and $0.5 \%$ BSA. The column was removed from the magnetic stand, and magnetically labeled cells were isolated by flushing out fractions. Total RNA was extracted from these isolated cells using TRIzol (Invitrogen) following the manufacturer's instructions.

qRT-PCR and allele-specific SNaPshot gene expression analysis. mRNA levels were measured by qRT-PCR as previously described (3). To determine strain-specific transcript levels between B6 and BALB/c, brain cortex from each inbred mouse strain was obtained at either P1 or $12 \pm 1$ week (adult). To quantify mRNA levels of $I l 21, I l 21 r$, Stat3, caspase 3, and Bcl2, brain slices from Il21r WT and $I l 21 r$ KO mice were used 0,12 , and 24 hours after OD exposure. All samples were run in triplicate, and an additional assay for endogenous Gapdh was performed to control for input cDNA template quantity.

Using brain cortex from $\mathrm{F} 1(\mathrm{~B} 6 \times \mathrm{BALB} / \mathrm{c})$ animals $(\mathrm{E} 18.5, \mathrm{P} 1$, and adult), cDNAs were generated and used to amplify regions of interest that included coding SNPs. Amplicons were generated by conventional PCR, and $15 \mu \mathrm{l}$ of PCR product was treated with $1 \mathrm{U}$ exonuclease I (New England Biolabs) and $5 \mathrm{U}$ of shrimp alkaline phosphatase (SAP) (Promega). Purified PCR products were used in an ABI SNaPshot assay (Thermo Fisher Scientific, catalog 4323159) according to the manufacturer's directions. These products were treated with $1 \mathrm{U}$ of SAP prior to electrophoresis on an ABI Prism 3130xl. Peak areas representing the SNP being queried were determined using GeneMapper software (ABI).

Collateral vessel density measurement. As collateral vessel traits are determined by 3 weeks of age and remain constant for many months (46), collateral phenotype was measured at P21. Mice were anesthetized with ketamine $(100 \mathrm{mg} / \mathrm{kg})$ and xylazine $(2.5 \mathrm{mg} / \mathrm{kg})$, and the ascending thoracic aorta was cannulated. The animals were perfused with freshly made buffer $(1 \mathrm{mg} / \mathrm{ml}$ adenosine, $40 \mathrm{~g} / \mathrm{ml}$ papaverine, and $25 \mathrm{mg} / \mathrm{ml}$ heparin in PBS) to remove the blood. The pial circulation was then exposed after removal of the dorsal calvarium and adherent dura mater. The cardiac left ventricle was cannulated and a polyurethane solution with a viscosity sufficient to minimize capillary transit (1:1 resin-to-2-butanone, PU4ii, VasQtec) was slowly infused; cerebral circulation was visualized under a stereomicroscope during infusion. The brain surface was topically rinsed with $10 \%$ PBS-buffered formalin and the dye solidified for 20 minutes. After post-fixation with $10 \%$ PBS-buffered formalin, pial circulation was imaged. All collaterals interconnecting the anterior and MCA trees of both hemispheres were counted.

Permanent MCAO. Focal cerebral ischemia was induced by direct permanent occlusion of the distal MCA as previously described (4). Briefly, adult mice were anesthetized with ketamine $(100 \mathrm{mg} / \mathrm{kg})$ and xylazine $(2.5 \mathrm{mg} / \mathrm{kg})$. The right MCA was exposed by a $0.5-\mathrm{cm}$ vertical skin incision midway between the right eye and ear under a dissecting microscope. After the temporalis muscle was split, a 2-mm burr hole was made with a high-speed microdrill at the junction of the zygomatic arch and the squamous bone through the outer surface of the semitranslucent skull. The MCA was clearly visible at the level of the inferior cerebral vein. The inner layer of the skull was removed with fine forceps, and the dura was opened with a 32-gauge needle.
While visualizing with an operating microscope, the right MCA was electrocauterized. The cauterized MCA segment was then transected with microscissors to verify permanent occlusion. The surgical site was closed with 6-0 sterile nylon sutures, and $0.25 \%$ bupivacaine was applied. The temperature of each mouse was maintained at $37^{\circ} \mathrm{C}$ with a heating pad during the surgery and until the animal was fully recovered from the anesthetic. Mice were then returned to their cages and allowed free access to food and water in an air-ventilated room with the ambient temperature set to $25^{\circ} \mathrm{C}$.

Transient MCAO and CBF. Transient MCAO was performed as described (47) previously, with minor modifications. Briefly, mice were anesthetized with $5 \%$ isoflurane in $30 \% \mathrm{O}_{2}$ balanced with $\mathrm{N}_{2}$. Then, mice were orally intubated and mechanically ventilated with $1.5 \%$ to $1.8 \%$ isoflurane. Rectal temperature was maintained at $37^{\circ} \mathrm{C}$ using a heating pad. A ventral neck incision was made, and the right carotid artery was dissected. Transient focal ischemia was induced by inserting a nylon monofilament (Doccol 602223) into the internal carotid artery along with ligation of the right common carotid artery. Ischemia was confirmed by laser-Doppler flowmetry (Moor) used to monitor regional CBF ( $\mathrm{rCBF}$ ) in the MCA cortex. After 45 minutes of occlusion, the monofilament was removed and the common carotid suture removed to allow MCA reperfusion and the incision was closed. Mice were given $0.5 \mathrm{ml}$ saline subcutaneously and then transferred into a temperature-controlled incubator for 2 hours before returning to their home cages.

Infarct volume measurement. Cerebral infarct volumes were measured 24 hours after surgery because the size of the cortical infarct is largest and is stable at 24 hours after distal permanent MCA occlusion (48). Twenty-four hours after MCAO surgery, the animals were euthanized by decapitation, and the brains were carefully removed. The brains were placed in a brain matrix and sliced into 1-mm coronal sections after being chilled at $-80^{\circ} \mathrm{C}$ for 3.5 minutes to slightly harden the tissue. Each brain slice was placed in 1 well of a 24 -well plate and incubated for 20 minutes in a solution of 2\% 2,3,5-triphenyltetrazolium chloride (TTC) in PBS at $37^{\circ} \mathrm{C}$ in the dark. The sections were then washed once with PBS and fixed with $10 \%$ PBS-buffered formalin at $4^{\circ} \mathrm{C}$. Then, 24 hours after fixation, the caudal face of each section was scanned using a flatbed color scanner. The scanned images were used to determine infarct volume (49). Image-Pro software (Media Cybernetics) was used to calculate the infarcted area of each slice by subtracting the infarcted area of the hemisphere from the noninfarcted area of the hemisphere to minimize error introduced by edema. The total infarct volume was calculated by summing the individual slices from each animal.

Preparation of brain slices. Preparation of cortical brain slice explants was performed as previously described $(18,19)$ with modifications. Neocortical brain slices were prepared from P10 mice from each strain. Under sterile conditions, the cerebral cortex was dissected and cut into $250-\mu \mathrm{m}$ coronal slices on a vibratome in chilled culture medium containing $15 \%$ heat-inactivated horse serum, $10 \mathrm{mM} \mathrm{KCl}, 10$ mM HEPES, $100 \mathrm{U} / \mathrm{ml}$ penicillin/streptomycin, $1 \mathrm{mM}$ MEM sodium pyruvate, and $1 \mathrm{mM}$ L-glutamine in Neurobasal A supplemented with NMDA receptor inhibitor (1 $\mu \mathrm{M}$ MK-801).

$O D$. The OD model was slightly modified from that we had previously established for OGD for inducing ischemic injury (18, 19). Unlike the OGD model, the OD model analyzes the same brain slices after stimulating ischemic injury by OD. Brain slices prepared 
as described above were placed into a 12-well plate with semisolid culture media $(0.5 \%$ agarose in brain slice preparation media without NMDA receptor inhibitor) and allowed to recover at $37^{\circ} \mathrm{C}$ for 30 minutes in a humidified incubator with $5 \% \mathrm{CO}_{2}$. Then, $1.6 \mu \mathrm{m}$ gold particle-coated plasmids encoding YFP were introduced into the brain slices by biolistic transfection using a Helios Gene Gun (BioRad, Hercules). After 24 hours of incubation, each slice was imaged to identify cortical pyramidal neurons expressing YFP, then submerged for 30 minutes at $37^{\circ} \mathrm{C}$ in $\mathrm{N}_{2}$-bubbled artificial CSF containing 140 $\mathrm{mM} \mathrm{NaCl}, 5 \mathrm{mM} \mathrm{KCl}, 1 \mathrm{mM} \mathrm{CaCl}, 1 \mathrm{mM} \mathrm{MgCl}, 24 \mathrm{mM}$ D-glucose, and $10 \mathrm{mM}$ HEPES to induce ischemic injury. The slices were carefully washed with prewarmed PBS and incubated for an additional 24 hours under normoxic conditions. At the end of this incubation time, the slices were reimaged. To assess cell viability, images taken before and after OD were compared by selecting a predetermined number of cortical pyramidal neurons in each slice at random without bias and the degree of overt neuronal degeneration and/or cell loss quantified by visual inspection. The experimenter performing the analysis was blinded to the treatment conditions.

Western blot analysis. To quantify protein expression levels, brain slices from Il21r KO mice, each inbred strain, and both congenic lines were collected either after OD treatment or after cytokine treatment. Apoptosis was determined by measuring apoptotic factor cleaved caspase 3 and antiapoptotic factor BCL-2. STAT3 phosphorylation was induced 1 hour after treatment with either recombinant IL-21 (50 $\mathrm{ng} / \mathrm{ml}$, PeproTech, catalog 210-21) or recombinant CNTF (80 ng/ $\mathrm{ml}$, PeproTech, catalog 450-50). Brain slices were homogenized in cold lysis buffer (50 mMTris- $\mathrm{HCl}$ [pH 7.8], $150 \mathrm{mM} \mathrm{NaCl}, 0.2 \%$ Triton $\mathrm{X}-100)$ containing protease and phosphatase inhibitor cocktail (Thermo Scientific, catalog 1861280). Protein samples (30-50 $\mu \mathrm{g})$ were electrophoresed on a $10 \%$ to $15 \%$ polyacrylamide gel and then transferred on PVDF membrane for 90 minutes on ice. Membranes were incubated with primary and secondary antibodies, and the level of protein was visualized via chemiluminescence (ECL Detection Kit, Thermo Fisher Scientific, 34080). The following primary antibodies were used: anti-cleaved caspase 3 (Asp175) (1:1,500, Cell Signaling Technology, 9661), anti-BCL-2 (50E3) (1:2,000, Cell Signaling Technology, 2870), anti-phospho-STAT3 (Tyr705) (1:1,500, Cell Signaling Technology, 9131), anti-STAT3 (C-20) (1:2,000, Santa Cruz Biotechnology Inc., sc-482), anti-GAPDH (1:4,000, Santa Cruz Biotechnol- ogy, sc-32233), and anti- $\alpha$-tubulin (1:7,500, Sigma-Aldrich, T6199). HRP-conjugated anti-mouse and anti-rabbit (Santa Cruz Biotechnology Inc.) secondary antibodies were used to detect proteins.

Immunohistochemistry. Tissue from ex vivo brain slice cultures was fixed overnight in $4 \%$ PFA and $4 \%$ sucrose at $4^{\circ} \mathrm{C}$. Brain slices were permeabilized and blocked with blocking solution containing $3 \%$ BSA and $0.2 \%$ Triton X-100 in PBS for 60 minutes at room temperature. Brain slices were incubated overnight with primary antibodies at $4^{\circ} \mathrm{C}$ and then incubated in secondary antibodies for 60 minutes at room temperature after washing with PBS. The following primary antibodies were used: anti-IL-21 (1:500, Abcam, ab13268), anti-IL-21R (1:500, Abcam, ab5978), and anti-MAP2 (1:1,000, SigmaAldrich, M1406). The following secondary antibodies were used: Alexa Fluor 488 and 594 (1:1,000, Molecular Probes). Images were captured using a Zeiss Axio Observer Z.1 microscope.

Statistics. Results are represented as the mean \pm SEM. Significant differences between data sets were determined using either a 2-tailed Student's $t$ test when comparing 2 groups or 1-way ANOVA followed by Scheffe's test for multiple comparisons. $P<0.05$ was considered significant.

Study approval. All animal study procedures were conducted with protocols approved by the Duke University IACUC in accordance with NIH guidelines.

\section{Author contributions}

HKL, DCL, and DAM designed research. HKL, SK, and HS performed research. HKL and DAM analyzed data. DCL and DSW contributed reagents. HKL, DCL, and DAM wrote the paper.

\section{Acknowledgments}

The authors thank Carol J. Gallione for critical comments and Jungki Min and Chul-Jin Lee for helpful discussion on IL-21R structure. This study was supported by the Holland-Trice Scholars Award, the Mandel Foundation, and NIH grant R01HL097281 (to D.A. Marchuk).

Address correspondence to: Douglas A. Marchuk, Department of Molecular Genetics and Microbiology, Box 3175, Duke University Medical Center, Durham, North Carolina 27710, USA. Phone: 919.684.1945; E-mail: douglas.marchuk@duke.edu.
1. Roger VL, et al. Heart disease and stroke statistics -2011 update: a report from the American Heart Association. Circulation. 2011;123(4):e18-e209.

2. Bevan S, et al. Genetic heritability of ischemic stroke and the contribution of previously reported candidate gene and genomewide associations. Stroke. 2012;43(12):3161-3167.

3. Keum S, et al. Natural genetic variation of integrin $\alpha \mathrm{L}$ (Itgal) modulates ischemic brain injury in stroke. PLoS Genet. 2013;9(10):e1003807.

4. Keum S, Marchuk DA. A locus mapping to mouse chromosome 7 determines infarct volume in a mouse model of ischemic stroke. Circ Cardiovasc Genet. 2009;2(6):591-598.

5. Chu PL, Keum S, Marchuk DA. A novel genetic locus modulates infarct volume independently of the extent of collateral circulation. Physiol Genomics. 2013;45(17):751-763.
6. Moskowitz MA, Lo EH, Iadecola C. The science of stroke: mechanisms in search of treatments. Neuron. 2010;67(2):181-198.

7. Zhang H, Prabhakar P, Sealock R, Faber JE. Wide genetic variation in the native pial collateral circulation is a major determinant of variation in severity of stroke. JCereb Blood Flow Metab. 2010;30(5):923-934.

8. Hossmann KA. Pathophysiology and therapy of experimental stroke. Cell Mol Neurobiol. 2006;26(7-8):1057-1083.

9. Dirnagl U, Simon RP, Hallenbeck JM. Ischemic tolerance and endogenous neuroprotection. Trends Neurosci. 2003;26(5):248-254.

10. Wang S, Zhang H, Dai X, Sealock R, Faber JE. Genetic architecture underlying variation in extent and remodeling of the collateral circulation. Circ Res. 2010;107(4):558-568.
11. Fantin A, et al. Tissue macrophages act as cellular chaperones for vascular anastomosis downstream of VEGF-mediated endothelial tip cell induction. Blood. 2010;116(5):829-840.

12. Ozaki K, et al. A critical role for IL-21 in regulating immunoglobulin production. Science. 2002;298(5598):1630-1634.

13. Spolski R, Leonard WJ. Interleukin-21: basic biology and implications for cancer and autoimmunity. Annu Rev Immunol. 2008;26:57-79.

14. Tzartos JS, et al. IL-21 and IL-21 receptor expression in lymphocytes and neurons in multiple sclerosis brain. Am J Pathol. 2011;178(2):794-802.

15. Kim YH, et al. Differential regulation of proliferation and differentiation in neural precursor cells by the Jak pathway. Stem Cells. 2010;28(10):1816-1828.

16. Wang $\mathrm{G}$, et al. Immunohistochemical localization 
of interleukin- 2 and its receptor subunits $\alpha, \beta$, and $\gamma$ in the main olfactory bulb of the rat. Brain Res. 2001;893(1-2):244-252.

17. Petitto JM, Huang Z, Raizada MK, Rinker CM, McCarthy DB. Molecular cloning of the cDNA coding sequence of IL-2 receptor-gamma (gammac) from human and murine forebrain: expression in the hippocampus in situ and by brain cells in vitro. Brain Res Mol Brain Res. 1998;53(1-2):152-162.

18. Dunn DE, He DN, Yang P, Johansen M, Newman RA, Lo DC. In vitro and in vivo neuroprotective activity of the cardiac glycoside oleandrin from Nerium oleander in brain slice-based stroke models. J Neurochem. 2011;119(4):805-814.

19. Wang JK, et al. Cardiac glycosides provide neuroprotection against ischemic stroke: discovery by a brain slice-based compound screening platform. Proc Natl Acad Sci U S A. 2006;103(27):10461-10466.

20. Hilfiker-Kleiner D, et al. Signal transducer and activator of transcription 3 is required for myocardial capillary growth, control of interstitial matrix deposition, and heart protection from ischemic injury. Circ Res. 2004;95(2):187-195.

21. Levy DE, Lee CK. What does Stat3 do? JClin Invest. 2002;109(9):1143-1148.

22. Takeda K, Akira S. Multi-functional roles of Stat3 revealed by conditional gene targeting. Arch Immunol Ther Exp (Warsz). 2001;49(4):279-283.

23. Wang $\mathrm{T}$, et al. Loss of interleukin-21 receptor activation in hypoxic endothelial cells impairs perfusion recovery after hindlimb ischemia. Arterioscler Thromb Vasc Biol. 2015;35(5):1218-1225.

24. Tauber MT, et al. Heterozygous mutation in the WSXWS equivalaent motif of the growth hormone receptor in a child with poor response to growth hormone therapy. Growth Horm IGF Res. 1998;8(3):211-216.

25. Hilton DJ, Watowich SS, Katz L, Lodish HF. Saturation mutagenesis of the WSXWS motif of the erythropoietin receptor. J Biol Chem. 1996;271(9):4699-4708.

26. Siupka P, Hamming OT, Kang L, Gad HH, Hartmann R. A conserved sugar bridge connected to the WSXWS motif has an important role for transport of IL-21R to the plasma membrane. Genes Immun. 2015;16(6):405-413.

27. Quelle DE, Quelle FW, Wojchowski DM. Mutations in the WSAWSE and cytosolic domains of the erythropoietin receptor affect signal transduction and ligand binding and internalization. Mol Cell Biol. 1992;12(10):4553-4561.

28. Hamming OJ, et al. Crystal structure of interleukin-21 receptor (IL-21R) bound to IL-21 reveals that sugar chain interacting with WSXWS motif is integral part of IL-21R. J Biol Chem. 2012;287(12):9454-9460.

29. Middleton G, et al. Cytokine-induced nuclear factor kappa B activation promotes the survival of developing neurons. JCell Biol. 2000;148(2):325-332.

30. Heinrich PC, Behrmann I, Müller-Newen G, Schaper F, Graeve L. Interleukin-6-type cytokine signalling through the gp130/Jak/STAT pathway. Biochem J. 1998;334(pt 2):297-314.

31. Taga T, Kishimoto T. Gp130 and the interleukin-6 family of cytokines. Annu Rev Immunol. 1997;15:797-819.

32. Leaman DW, Leung S, Li X, Stark GR. Regulation of STAT-dependent pathways by growth factors and cytokines. FASEB J. 1996;10(14):1578-1588.

33. Parrish-Novak J, et al. Interleukin 21 and its receptor are involved in NK cell expansion and regulation of lymphocyte function. Nature. 2000;408(6808):57-63.

34. Rodríguez-Bayona B, Ramos-Amaya A, Bernal J, Campos-Caro A, Brieva JA. Cutting edge: IL-21 derived from human follicular helper $\mathrm{T}$ cells acts as a survival factor for secondary lymphoid organ, but not for bone marrow, plasma cells. JImmunol. 2012;188(4):1578-1581.

35. Leonard WJ, Zeng R, Spolski R. Interleukin 21: a cytokine/cytokine receptor system that has come of age. J Leukoc Biol. 2008;84(2):348-356.

36. Zeng R, et al. Synergy of IL-21 and IL-15 in regulating $\mathrm{CD}^{+}{ }^{+} \mathrm{T}$ cell expansion and function. J Exp Med. 2005;201(1):139-148.

37. Dokun AO, et al. A quantitative trait locus (LSq-1) on mouse chromosome 7 is linked to the absence of tissue loss after surgical hindlimb ischemia. Circulation. 2008;117(9):1207-1215.
38. Kotlarz D, et al. Loss-of-function mutations in the IL-21 receptor gene cause a primary immunodeficiency syndrome. JExp Med. 2013;210(3):433-443.

39. Wilson RP, et al. STAT3 is a critical cell-intrinsic regulator of human unconventional $\mathrm{T}$ cell numbers and function. JExp Med. 2015;212(6):855-864.

40. Pesce J, et al. The IL-21 receptor augments Th2 effector function and alternative macrophage activation. JClin Invest. 2006;116(7):2044-2055.

41. Hossmann KA. The two pathophysiologies of focal brain ischemia: implications for translational stroke research.J Cereb Blood Flow Metab. 2012;32(7):1310-1316.

42. Clarkson BD, et al. T cell-derived interleukin (IL)21 promotes brain injury following stroke in mice. J Exp Med. 2014;211(4):595-604.

43. Lo EH, Dalkara T, Moskowitz MA. Mechanisms, challenges and opportunities in stroke. Nat Rev Neurosci. 2003;4(5):399-415.

44. Yao H, Cui ZH, Masuda J, Nabika T. Congenic removal of a QTL for blood pressure attenuates infarct size produced by middle cerebral artery occlusion in hypertensive rats. Physiol Genomics. 2007;30(1):69-73.

45. Jeffs B, et al. Sensitivity to cerebral ischaemic insult in a rat model of stroke is determined by a single genetic locus. Nat Genet.1997;16(4):364-367.

46. Clayton JA, Chalothorn D, Faber JE. Vascular endothelial growth factor-A specifies formation of native collaterals and regulates collateral growth in ischemia. Circ Res. 2008;103(9):1027-1036.

47. Sheng $\mathrm{H}$, et al. Apolipoprotein $\mathrm{E}$ isoform-specific differences in outcome from focal ischemia in transgenic mice. J Cereb Blood Flow Metab. 1998;18(4):361-366.

48. Lambertsen KL, et al. A quantitative study of microglialmacrophage synthesis of tumor necrosis factor during acute and late focal cerebral ischemia in mice. J Cereb Blood Flow Metab. 2005;25(1):119-135.

49. Wexler EJ, Peters EE, Gonzales A, Gonzales ML, Slee AM, Kerr JS. An objective procedure for ischemic area evaluation of the stroke intraluminal thread model in the mouse and rat. J Neurosci Methods. 2002;113(1):51-58. 